\title{
Neutron spin structure studies at EIC
}

\author{
Alessio Del Dotto* \\ INFN Laboratori Nazionali di Frascati, Italy \\ E-mail: alessio.deldotto@lnf.infn.it
}

\section{Evaristo Cisbani, Giovanni Salmè}

INFN Rome, Italy

\section{Leonid Kaptari}

Bogoliuboc Laboratory of Theoretical Physics, Russia

\section{Emanuele Pace}

University of Rome 'Tor Vergata' and INFN Roma Tor Vergata, Italy

\section{Sergio Scopetta}

Perugia University and INFN Perugia, Italy

\begin{abstract}
The extended and precise investigation of the nucleon (spin) structure is a cornerstone of the EIC initiative; the availability of deuteron and ${ }^{3} \mathrm{He}$ polarized beams will offer unprecedented opportunities to obtain information on the neutron spin structure comparable to those of the proton; the combination of these data will allow to deepen knowledge and understanding of many aspects of the nucleon spin physics: from a more precise test of the Bjorken sum rule to a more detailed picture of the flavor structure of the nucleon sea, as well as a precise determination of different spin and transverse momentum dependent functions, at leading and higher twists. Moreover, almost close kinematics and large acceptance detectors typical of a collider, will potentially offer new measurement perspectives beyond the asymmetries.
\end{abstract}

23rd International Spin Physics Symposium - SPIN2018 -

10-14 September, 2018

Ferrara, Italy

\footnotetext{
*Speaker.
} 


\section{Introduction}

The study of the nucleon intricate inner dynamics strongly demand experimental inputs from both isospin states: proton and neutron. While proton data set is relatively extended [1], the current experimental investigations of the spin structure of the neutron is penalized by the impossibility of producing an exploitable free neutron polarized target, requiring the use of "mixed" target containing neutrons: e.g. deuteron [2] and ${ }^{3} \mathrm{He}$ : while in $2 H, p$ and $n$ have similar contributions, both in terms of interactions and spin, ${ }^{3} \mathrm{He}$ has a more complex nuclear effects on the $n$, but it is an almost pure neutron polarized target (namely, the two protons are almost in wave $S=0$ ). The two targets are complementary and both are actually needed to extract consistent data on neutron from non-trivial nuclear effects.

New experiments are under data taking or planned in the coming years on ${ }^{3} \mathrm{He}$ (un)polarized (and the mirror ${ }^{3} \mathrm{H}$ ) fixed target $[11,12,13]$, which will provide precise multidimensional (polarized) (SI)DIS data at large $0.1<x_{B j}<0.7$ (and moderate $1<Q^{2}<10 \mathrm{GeV}^{2}$ ) together with experiments on H/D based targets.

In this respect, the Electron-Ion Collider (EIC) will offer unique opportunities in the next decade, as discussed in many presentations of this conference, with particular reference to [2] for deuteron and the present paper for ${ }^{3} \mathrm{He}$ : the boost of the ion beam favorites the investigation of the spectator target technique not only on the deuteron but also on ${ }^{3} \mathrm{He}$, as discussed in the following. Moreover the maximum high energy $(\sqrt{s} \sim 50 \mathrm{GeV})$ combined to a foreseen luminosity at the level of $10^{34} / \mathrm{cm}^{2} / \mathrm{s}$ will permit the exploration of a large $\left(x_{B j}, Q^{2}\right)$ phase space $\left(x_{B j}\right.$ down to $\left.10^{-4}\right)$, with adequate statistics.

\section{Neutron spin structure status}

Latest measurements on lower twist neutron Transverse Momentum dependent Distribution functions (TMDs), have been collected on SIDIS off ${ }^{3} \mathrm{He}$ transversely polarized target at Thomas Jefferson Laboratory (JLab) $[3,4]$ in the $x_{B j}$ valence region; the remarkably different from zero $A_{L T}$ asymmetries with opposite signs for the pions, but with limited data, and the negligible Sivers and Collins TMDs for $K$ and $\pi$, except a negative Sivers for $\pi^{+}$, not fully consistent with the $p$ and $D$ existing data, do not present a clear picture but demand for further experimental data on a broader phase space and with higher precision. Moreover lower twist "simple" models seem to reproduce reasonably well the main features of the present ${ }^{3} \mathrm{He}$ unpolarized cross sections data in the valence region [5] while the model dependent width of intrinsic transverse momentum $K_{T}^{2}$ is sizable lower than the width coming from global analyses. It is worth mentioning that cross section (polarized) data are very valuable to perform quantitative estimate of the transverse quark motion and the FSI in light nuclei [6]. In this respect, the two colliding beams of EIC, combined to hermetic, almost $4 \pi$, detector offers advantages respect to fixed target, limited acceptance experiments.

\section{3. ${ }^{3}$ He polarized effective neutron target: towards a complete description of the system}

A polarized ${ }^{3} \mathrm{He}$ nucleus is an ideal target to study the neutron, since at a $90 \%$ level it is equivalent to a polarized neutron. For disentangling the nucleon structure from the dynamical 
nuclear effects, one can adopt an approach primarily based on the spin-dependent spectral function of ${ }^{3} \mathrm{He}, \mathrm{P}_{\sigma, \sigma^{\prime}}(\vec{p}, E)$, (see, e.g. [7]) that yields the probability distribution to find a nucleon with given missing energy (the spectator pair is interacting), three-momentum and polarization inside the nucleus. By using this formalism, known as Plane Wave Impulse Approximation (PWIA), one can safely extract [9] the neutron longitudinal asymmetry, $A_{n}$, from the corresponding ${ }^{3} \mathrm{He}$ observable, $A_{3}^{\text {exp }}$, obtained from the reaction ${ }^{3} \mathrm{He}\left(\vec{e}, e^{\prime}\right) X$ in DIS regime, i.e.

$$
A_{n} \simeq\left(A_{3}^{\exp }-2 p_{p} f_{p} A_{p}^{\exp }\right) /\left(p_{n} f_{n}\right)
$$

with $p_{n(p)}$ the neutron (proton) effective polarization inside the polarized ${ }^{3} \mathrm{He}$, and $f_{n(p)}$, the dilution factor. Realistic values of $p_{n}$ and $p_{p}$ are $p_{p}=-0.023, p_{n}=0.878$ (see, e.g., [8, 9]). In [9], an analogous extraction was applied to the SSA of a transversely polarized ${ }^{3} \overrightarrow{\mathrm{He}}$ target, obtained from the process ${ }^{3} \mathrm{He}\left(e, e^{\prime} \pi\right) X$, in order to obtain the SSA of a transversely polarized neutron. In PWIA and adopting the Bjorken limit, the SSAs of ${ }^{3} \overrightarrow{\mathrm{He}}$ are a convolution of $\mathrm{P}_{\sigma, \sigma^{\prime}}(\vec{p}, E)$, and the nucleon SSAs, that in turn are convolutions of suitable TMDs and fragmentation functions (FF), phenomenologically describing the hadronization of the hit quark.

The main ingredients for the extraction where: i) a realistic $\mathrm{P}_{\sigma, \sigma^{\prime}}(\vec{p}, E)$ for the ${ }^{3} \mathrm{He}$, obtained using the AV18 interaction; ii) parametrizations of data for TMDs and FFs, whenever available; iii) models for the unknown TMDs and FFs. Within this framework, the extraction formula (3.1) works well even in the case of SIDIS, at least at the present statistical accuracy of the existing data. Indeed Eq. (3.1) has been used by the JLab Hall A Collaboration to extract, for the first time, the Collins and Sivers moments from a transversely polarized ${ }^{3} \mathrm{He}$ target [3]. The expected statistical accuracy for the future programs is of the order of percent in a wide range of multi-dimensional kinematics binning; for this reason the PWIA could be no longer sufficient and the FSI, (not considered in PWIA) may have a non-negligible role.

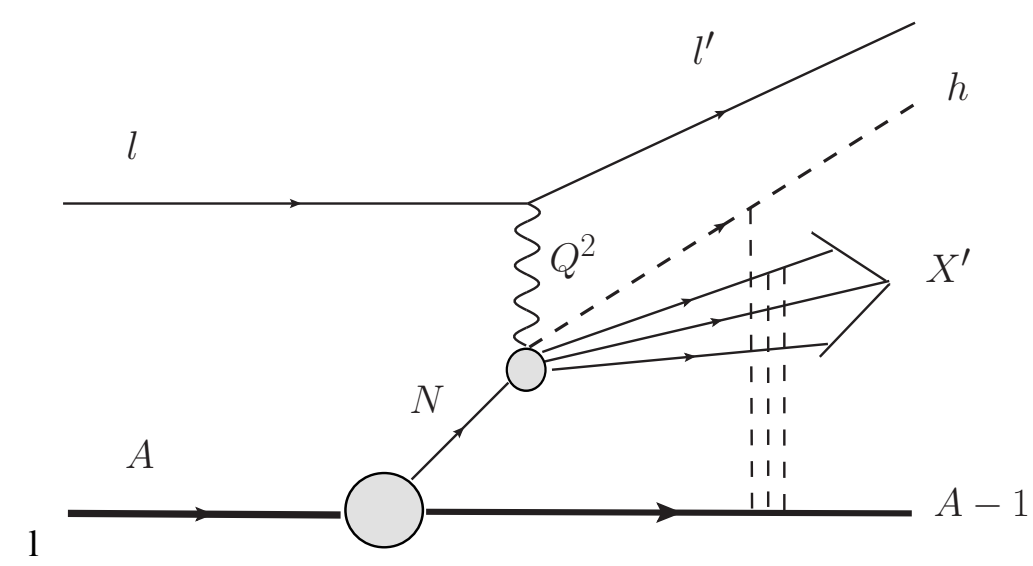

Figure 1: Interaction between the $(A-1)$ spectator system (fully interacting) and the debris produced by the absorption of a virtual photon by a nucleon in the nucleus.

Recently a distorted spin-dependent spectral function of the ${ }^{3} \mathrm{He}$ has been calculated $[6,10]$, which includes FSI treated within a generalized eikonal approximation framework (GEA). It has been calculated how the polarized SIDIS cross section depends on a convolution of the spectral function with the TMDs entering in a given combination of quark-nucleon polarizations; namely 
the key quantity is the light-cone distribution function of the ${ }^{3} \mathrm{He}$ (see Fig. 2). FSI effects have been found to produce sizable effects in both the unpolarized and polarized cross sections. Differently, the SSAs have resulted slightly affected by FSI, since they are ratio of cross sections, and therefore the FSI effects cancel to a large extent [10]. As a result, in the kinematical range relevant for ongoing SIDIS experiments (i.e. at JLab), the very same extraction procedure proven to be successful in PWIA can be used also in a scenario where FSI effects are relevant. Nevertheless two aspects can be further underlined: i) The relative difference between the PWIA and FSI model predictions at the peak is about 50\%; ii) the correspondent difference in the mean polarization is of the same order. This open an interesting opportunity in studying FSI by measuring asymmetries or (even more sound) cross sections in appropriate kinematical regions. In the following we will emphasize an application to the so called spectator SIDIS, namely the semi-inclusive process in which the scattered electron is detected in coincidence with the $A-1$ system.

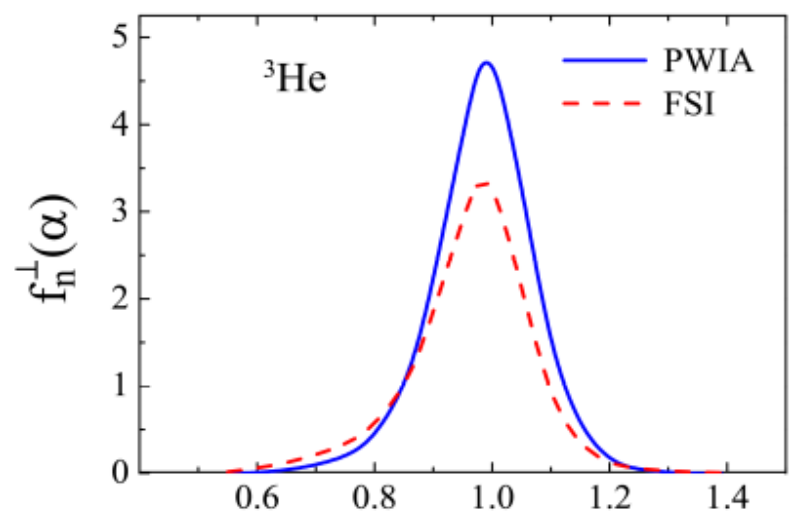

$\alpha$

Figure 2: The neutron transversely polarized distribution as a function of the light cone momentum fraction $\alpha$, from [10].

\section{Spectator SIDIS in ${ }^{3} \mathrm{He}$}

The relevant components of the above mentioned spin-dependent distorted spectral function have been applied to the SIDIS processes with polarized particles $[6,10]$. The single spin asymmetry involving the most known spin dependent structure function $g_{1}^{N}$ can be written as:

$$
\begin{aligned}
& \frac{\Delta \sigma^{\hat{\mathbf{S}}_{A}}}{d \varphi_{e} d x_{B j} d y d \mathbf{P}_{D}} \equiv \frac{d \sigma^{\hat{\mathbf{S}}_{A}}\left(h_{e}=1\right)-d \sigma^{\hat{\mathbf{S}}_{A}}\left(h_{e}=-1\right)}{d \varphi_{e} d x_{B j} d y d \mathbf{P}_{D}} \\
& \approx 4 \frac{\alpha_{e m}^{2}}{Q^{2} z_{N} \mathscr{E}} \frac{m_{N}}{E_{N}} g_{1}^{N}\left(\frac{x_{B j}}{z_{N}}\right) \mathscr{P}_{\|}^{\frac{1}{2}}\left(\mathbf{p}_{m i s}\right) \mathscr{E}(2-y)\left[1+\frac{\left|\mathbf{p}_{m i s}\right|}{m_{N}}\right]
\end{aligned}
$$

The variable $z_{N}$ is proportional to the light-cone fraction of the momentum carried by the nucleon in the nucleus, i.e. $z_{N}=\left(p_{N} \cdot q\right) / m_{N} v$; it yields a measure of the difference between $x_{B j}$ and $x_{N}$, that is the actual variable upon which $g_{1}^{N}$ depends (for more details see [6]). It is important to point out that: 
- this asymmetry (and also the related cross sections) are a convolution of $g_{1}^{N}$ with the ${ }^{3} \mathrm{He}$ spectral function $\mathscr{P}_{\|}^{\frac{1}{2}}$;

- measuring a deuteron in the spectator (A-1) system will provide a measurement of $g_{1}^{p}$ and if compared with the same observable from proton target this will give access to a measurement of FSI;

- at the same time a better knowledge of $g_{1}^{p}$ will allow a better knowledge of the EMC effect in this system;

- the measurement of a $p p$ state in the $A-1$ system will allow the extraction of $g_{1}^{n}$.

As already mentioned the EIC collider beams provide a favorable phase space for the investigation of semi-exclusive processes: $e+{ }^{3} \mathrm{He} \rightarrow e^{\prime}+\varsigma+\pi$ (or $\left.K\right)+X$, with $\varsigma$ the very forward detected spectator ( $\mathrm{D}$ or $\mathrm{pp}$ ), and $X$ the undetected particle(s). In fact the momentum of the spectator $p_{\varsigma} \simeq 2 / 3 \cdot p^{3} \mathrm{He}$ is almost collinear to the ion beam, requiring a very forward detector.
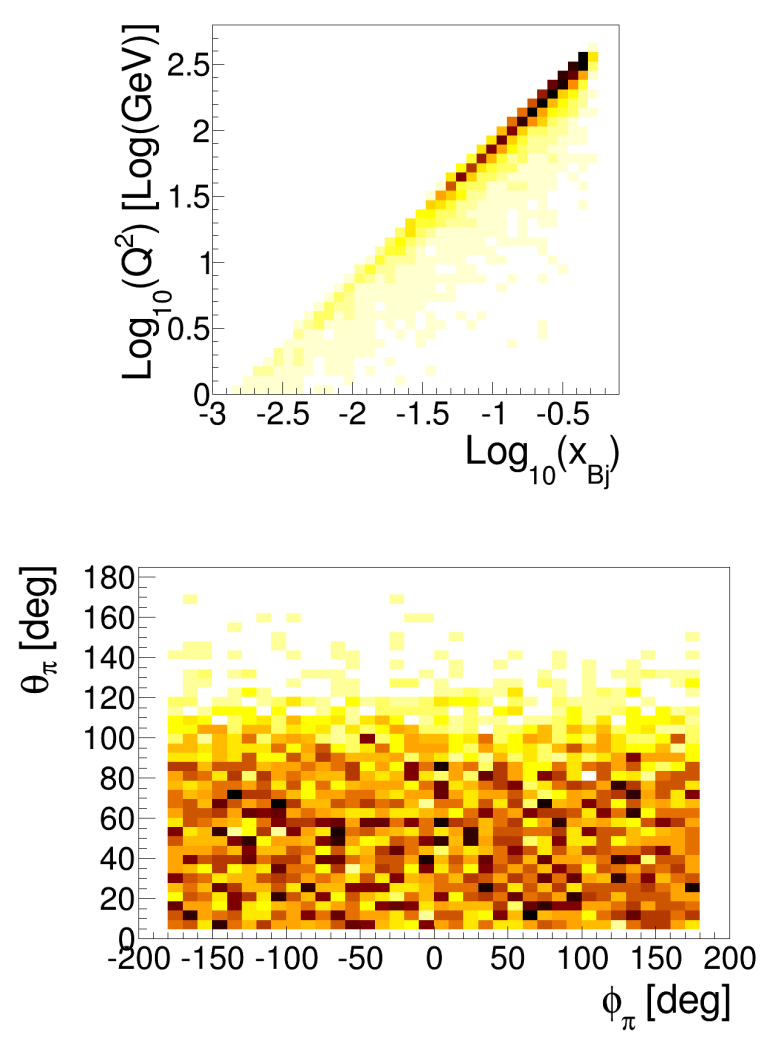
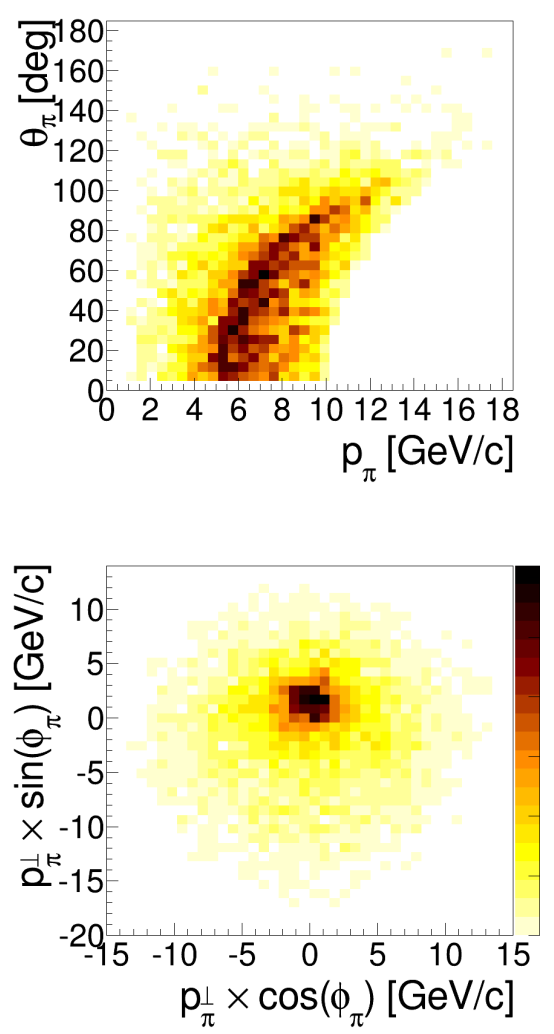

Figure 3: DIS variables, absolute value $(p)$, polar $(\theta)$ and azimuth $(\phi)$ angles of the detected hadron $(\pi)$ momentum reported in their correlated phase spaces; angles are respect to the direction of the exchanged photon momentum. Distributions correspond to electron and ${ }^{3} \mathrm{He}$ beams incidence momenta of $10 \mathrm{GeV}$ and $60 \mathrm{GeV}$ respectively.

From the spectator momentum one can reconstruct the invariant mass of the initial nucleon $(N): t=\left(p^{3} \mathrm{He}_{\mathrm{e}}-p_{\varsigma}\right)^{2} \simeq m_{N}^{2}$. Figures 3 and 4 shows the phase spaces of some of the most relevant 

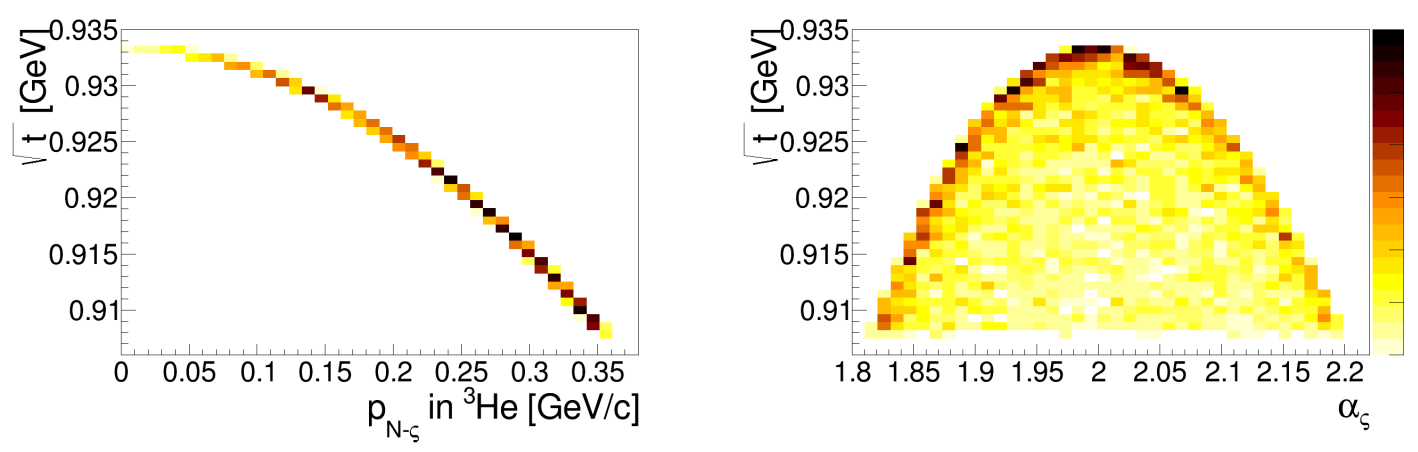

Figure 4: (left panel) Initial neutron momentum $p_{N-\varsigma}$ in ${ }^{3} \mathrm{He}$ and (right panel) invariant momentum transfer between ion target and recoil spectator versus the light-cone momentum fraction of the spectator $\alpha_{\varsigma}=$ $A\left(p_{\varsigma} \cdot q\right) /\left(P_{A} \cdot q\right)$; momentum unit is GeV. On-shellness of the initial neutron is guaranteed by $\sqrt{t} \sim m_{N}$, where the largest fraction of phase space seats.

variables of the above reaction, assuming electron and ${ }^{3} \mathrm{He}$ beam incidence momenta of $10 \mathrm{GeV}$ and $60 \mathrm{GeV}$ respectively. The angular and momentum acceptances of the detected particles $(\pi$ and $\varsigma$ ) are consistent with most of the current EIC spectrometers and interaction point proposed configurations [14]. An analysis of the achievable (and desirable) uncertainties is required; it may further constraint the detector specifications, especially for the very forward spectrometer that shall detect the spectator nucleons; however these preliminary distributions confirm the important impact the ${ }^{3} \mathrm{He}$ SIDIS-spectator technique has on the investigation of the structure of the neutron, including the extraction of the transverse momentum distributions, e.g. through azimuthal analysis (bottom plots of figure 3 show an optimal angular coverage), with unprecedented control on the initial conditions of the partons (figure 4).

\section{Conclusions}

The experimental neutron (spin) structure functions are intrinsically involved with nuclear effects due to the impossibility to have a free neutron target. To "clearly" get rid of the nuclear effects, we believe that at least, Deuteron and ${ }^{3} \mathrm{He}$ targets need to be experimentally investigated at EIC; both will provide complementary information on the neutron structure and at the same time valuable information on their own internal dynamics. In fact theoretical approaches are under refinement and extension for both FSI and other low $x_{B j}$ effects (e.g. anti-shadowing) in ${ }^{3} \mathrm{He}$. Measurement of (polarized) cross section, in addition to asymmetries, may open interesting scenarios in understanding the nuclear effects; good luminosity monitor and detector performances are required.

The almost exclusive (polarized) DIS through the spectator technique is expected to be applicable to ${ }^{3} \mathrm{He}$ as well as to the $\mathrm{D}$ spectator tagging; investigation of such reactions may represent a valuable step forward in EIC, although the costruction of a ${ }^{3} \mathrm{He}$ adequate polarimetry is not trivial in a collider and need a careful research and development. 


\section{References}

[1] C.A. Aidala et al. "The spin structure of the nucleon." Reviews of Modern Physics 85.2 (2013): 655.

[2] Cosyn, W. "DIS on a polarized deuteron with spectator nucleon tagging", this Proceedings.

[3] Qian, X. et al. "Single Spin Asymmetries in Charged Pion Production from Semi-Inclusive Deep Inelastic Scattering on a Transversely Polarized ${ }^{3} \mathrm{He}$ Target at $Q^{2}=1.42 .7 \mathrm{GeV}^{2}$ ", PRL 107, 072003 (2011)

[4] Zhao, Y. X. et al. "Double spin asymmetries of inclusive hadron electroproduction from a transversely polarized ${ }^{3}$ He target”, Phys. Rev. C 92015207 (2015)

[5] Yan, X. et al. "First measurement of unpolarized semi-inclusive deep-inelastic scattering cross sections from a He 3 target”, Physical Review C 95.3 (2017): 035209.

[6] Kaptari, L. P., Del Dotto, A., Pace, E., Salmè, G., Scopetta, S. (2014). "Distorted spin-dependent spectral function of an $\mathrm{A}=3$ nucleus and semi-inclusive deep-inelastic scattering processes" Physical Review C, 89(3), 035206.

[7] Ciofi degli Atti, C., Pace, E. and Salmè, G., "Spin dependent spectral function of ${ }^{3} \mathrm{He}$ and the asymmetry in the process ${ }^{3} \mathrm{He}(e, e) X$ ”, Phys. Rev. C 46, 1591 (1992).

[8] Ciofi degli Atti, C., Pace, E., Salmè, G. and Scopetta, S., "Nuclear effects in deep inelastic scattering of polarized electrons off polarized ${ }^{3} \mathrm{He}$ and the neutron spin structure functions", Phys. Rev. C 48, R968 (1993).

[9] Scopetta, S. "Neutron single spin asymmetries from semi-inclusive deep inelastic scattering off transversely polarized ${ }^{3} \mathrm{He}$ ", Phys. Rev. D 75, 054005 (2007).

[10] Del Dotto, A., Kaptari, L. P., Pace, E., Salmè, G., Scopetta, S. (2017). "Final state interactions and the extraction of neutron single spin asymmetries from semi-inclusive deep-inelastic scattering by a transversely polarized ${ }^{3}$ He target”, Physical Review C, 96(6), 065203.

[11] G. Petratos et al., "Measurement of $F_{2 n} / F_{2 p}, \mathrm{~d} / \mathrm{u}$ ratios and $A=3$ EMC effect in deep inelastic electron scattering of the Tritium and Helium mirror nuclei”, JLab Exp. E12-10-103;

[12] Cates, G. et al., "Measurement of Semi-inclusive Pion and Kaon Electroproduction on a Trasversely Polarized ${ }^{3}$ He target", JLab Exp. E12-09-018;

[13] Zhao, Z. "Nucleon 3D imaging program with SoLID at Jefferson Lab", this Proceedings.

[14] P. Nadel-Turonski, "Opportunities with light ions at the EIC", this Proceedings. 